\title{
Multisynaptic projections from the ventrolateral prefrontal cortex to hand and mouth representations of the monkey primary motor cortex.
}

\section{$\operatorname{AUTHOR}(S)$ :}

Miyachi, Shigehiro; Hirata, Yoshihiro; Inoue, KenIchi; Lu, Xiaofeng; Nambu, Atsushi; Takada, Masahiko

\section{CITATION:}

Miyachi, Shigehiro ...[et al]. Multisynaptic projections from the ventrolateral prefrontal cortex to hand and mouth representations of the monkey primary motor cortex..

Neuroscience research 2013, 76(3): 141-149

\section{ISSUE DATE:}

2013-07

URL:

http://hdl.handle.net/2433/175588

\section{RIGHT:}

(C) 2013 Elsevier Ireland Ltd and the Japan Neuroscience Society.; この 論文は著者最終稿です。内容が印刷版と異なることがありますので、 引用の際には出版社版をご確認ご利用ください。This is the Accepted Author Manuscript. Please cite only the published version. 
Title: Multisynaptic projections from the ventrolateral prefrontal cortex to hand and mouth representations of the monkey primary motor cortex

Authors: Shigehiro Miyachi ${ }^{1}$, Yoshihiro Hirata ${ }^{2}$, Ken-ichi Inoue ${ }^{3}$, Xiaofeng $\mathrm{Lu}^{4,5}$, Atsushi Nambu ${ }^{6}$, Masahiko Takada ${ }^{3}$

\section{Addresses:}

${ }^{1}$ Cognitive Neuroscience Section, Primate Research Institute, Kyoto University, Inuyama 484-8506, Japan

${ }^{2}$ Photonic Bioimaging Section, Research Center for Cooperative Projects, Hokkaido University Graduate School of Medicine, Sapporo 060-8638, Japan

${ }^{3}$ Systems Neuroscience Section, Primate Research Institute, Kyoto University, Inuyama 484-8506, Japan

${ }^{4}$ Brain Sciences Center, VA Medical center, Minneapolis, MN 55417, USA

${ }^{5}$ Department of Neurology, University of Minnesota School of Medicine, Minneapolis, MN 55414, USA

${ }^{6}$ Division of System Neurophysiology, National Institute for Physiological Sciences and Department of Physiological Sciences, Graduate University for Advanced Studies, Okazaki 444-8585, Japan

Corresponding author: S. Miyachi, Cognitive Neuroscience Section, Primate Research Institute, Kyoto University, 41-2 Kanrin, Inuyama, Aichi 484-8506, Japan. E-mail: miyachi.shigehiro.8e@kyoto-u.ac.jp 
Tel: +81-568-63-0559 Fax: +81-568-63-0563

Number of pages 34; Number of figures 5; Number of table 1; Number of supplementary figure 1. 


\section{Abstract}

Different sectors of the prefrontal cortex have distinct neuronal connections with higher-order sensory areas and/or limbic structures and are related to diverse aspects of cognitive functions, such as visual working memory and reward-based decision-making. Recent studies have revealed that the prefrontal cortex (PF), especially the lateral PF, is also involved in motor control. Hence, different sectors of the PF may contribute to motor behaviors with distinct body parts. To test this hypothesis anatomically, we examined the patterns of multisynaptic projections from the PF to regions of the primary motor cortex (MI) that represent the arm, hand, and mouth, using retrograde transsynaptic transport of rabies virus. Four days after rabies injections into the hand or mouth region, particularly dense neuron labeling was observed in the ventrolateral PF, including the convexity part of ventral area 46. After the rabies injections into the mouth region, another dense cluster of labeled neurons was seen in the orbitofrontal cortex (area 13). By contrast, rabies labeling of PF neurons was rather sparse in the arm-injection cases. The present results suggest that the PF-MI multisynaptic projections may be organized such that the MI hand and mouth regions preferentially receive cognitive information for execution of elaborate motor actions.

Key words: transneuronal tracing; rabies virus, area 46; area 12; motor control; somatotopy; forelimb; orofacial. 


\section{Introduction}

The prefrontal cortex is composed of several anatomically distinct areas which have different neuronal connections with the posterior association cortices and the limbic-related structures (for review, Pandya and Yeterian, 1990). According to such diverse connectivity, different prefrontal areas exert control influences on behaviors in different ways. The dorsolateral and ventrolateral prefrontal cortices (DLPF and VLPF) receive strong inputs from the visual association areas of the parietal and temporal cortices (Barbas and Mesulam, 1985; Barbas, 1988; Petrides and Pandya, 1999, 2001), respectively and, consequently play key roles in working memory and decision making based on spatial vs. nonspatial visual information (Passingham, 1975; Mishkin and Manning, 1978; Wilson et al., 1993; Miller et al., 1996; Warden and Miller, 2007). On the other hand, the orbitofrontal cortex (OF) has dense neuronal connections with limbic structures as well as with the sensorimotor cortical areas (Barbas, 1993; Carmichael and Price, 1995a, b), and has been implicated in reward-based decision-making (Tremblay and Schultz, 1999, 2000; Hikosaka and Watanabe, 2000, 2004; Bouret and Richmond, 2010).

Besides the activities related to the working-memory and decision-making processes, the prefrontal neurons display activities related to eye and somatic movements. Regarding the somatic movements, di Pellegrino and Wise (1991) found that many neurons in the VLPF, rather than the DLPF, showed motor-related activities in a matching-to-sample task. Hoshi et al. (1998) also recorded many motor-related neuron activities in the VLPF, including the lip of the principal sulcus and the ventral convexity.

In accordance with the existence of motor-related activities, this prefrontal area 
has intracortical connections with the motor-related areas, such as the cingulate motor and premotor areas and the ventral bank of the intraparietal sulcus (Barbas, 1988; Petrides and Pandya, 2001; Luppino et al., 2003). Luppino and his colleagues emphasized that a VLPF region, including ventral area 46 (area 46v) and rostral area 12 (area 12r), has specific connections with the rostral part of the ventral premotor cortex and the anterior intraparietal area (AIP) (Gerbella et al., 2010, 2011, 2012; Borra et al., 2011). They proposed that this region of the VLPF is involved in the control of hand and mouth actions based on non-spatial visual information.

We have previously examined the multisynaptic projections from the prefrontal cortex to regions of the primary motor cortex (MI) that represent the forelimb (including both the arm and the hand) and hindlimb, using rabies virus as a retrograde transsynaptic tracer (Miyachi et al., 2005). We found that the MI forelimb region receives much stronger inputs from the prefrontal cortex than the hindlimb region. Especially, we have defined that, among the prefrontal areas, the VLPF is the major origin of these prefrontal inputs to the MI forelimb region.

In the present study, we focused on the prefrontal inputs to MI regions representing the hand and mouth, as these two effectors contribute to the most elaborate motor actions. Rabies virus was injected separately into hand (wrist/digits) and mouth (jaw/lip/tongue) representations of the MI, and the patterns of retrograde transneuronal labeling in the prefrontal cortex were compared with that in the case of rabies injections into arm (shoulder/elbow) representation. 


\section{Materials and Methods}

Six adult macaque monkeys ( 3 rhesus, 2 Japanese, and 1 crab-eating monkeys) of either sex were used in the present study (Table 1). In monkeys $\mathrm{X}, \mathrm{Lo}, \mathrm{Su}, \mathrm{Si}$, and $\mathrm{Ch}$, data on neuronal labeling in the basal ganglia were separately reported elsewhere (Miyachi et al., 2006). The experimental protocols were approved by the Animal Care and Use Committee of the Tokyo Metropolitan Institute for Neuroscience. All experiments were carried out according to the Guideline for the Care and Use of Animals (Tokyo Metropolitan Institute for Neuroscience 2000).

\section{Surgical Procedures and Electrophysiological Mapping}

All surgical and electrophysiological procedures have been fully described in our prior papers (Miyachi et al., 2005, 2006). Briefly, under general anesthesia with ketamine hydrochloride (5-10 mg/kg, i.m.), xylazine hydrochloride $(0.5-1 \mathrm{mg} / \mathrm{kg}$, i.m.), and sodium pentobarbital $(20 \mathrm{mg} / \mathrm{kg}$, i.v.), a head holder was attached to the exposed skull of each monkey in aseptic conditions. Several days later, arm (shoulder/elbow), hand (wrist/digits), or mouth (jaw/lip/tongue) representation in the MI was mapped in individual monkeys by electrophysiological methods. The monkeys were anesthetized 
with a combination of ketamine hydrochloride (5-10 $\mathrm{mg} / \mathrm{kg}$, i.m.) and xylazine hydrochloride $(0.5-1 \mathrm{mg} / \mathrm{kg}, \mathrm{i} . \mathrm{m}$.$) and seated in a primate chair with their head fixed$ painlessly in a stereotaxic frame attached to the chair. After partial removal of the skull over the frontal cortex, a glass-coated Elgiloy-alloy microelectrode (0.5-1.5 $\mathrm{M} \Omega$ at 1 $\mathrm{kHz}$ ) was introduced perpendicular to the dural surface. Intracortical microstimulation (ICMS; trains of 12 cathodal pulses, $200 \mu \mathrm{sec}$ duration at $333 \mathrm{~Hz}$, less than $50 \mu \mathrm{A}$ ) was applied, and evoked movements of various body parts were carefully monitored by visual inspection. Then, a chamber made of polyacetal was attached to the skull with acrylic resin for later rabies virus injections.

\section{Rabies Virus Injections}

The CVS-11 strain of rabies virus was derived from the Center for Disease Control and Prevention (Atlanta, GA, USA) and donated by Dr. Satoshi Inoue of the National Institute of Infectious Diseases (Tokyo, Japan). Under the anesthesia with ketamine hydrochloride (5-10 mg/kg, i.m.) and xylazine hydrochloride (0.5-1 mg/kg, i.m.), viral suspension (1.4 $\mathrm{X} 10^{8}$ focus-forming units $/ \mathrm{ml}$ ) was injected into the electrophysiologically identified MI regions $(0.5-1 \mu \mathrm{l} /$ penetration, $6-10$ penetrations) through a $10-\mu 1$ Hamilton microsyringe 
based on the somatotopic mapping: arm (shoulder and elbow) region in monkeys $\mathrm{X}$ and Lo, hand (wrist and digits) region in monkeys $\mathrm{Su}$ and $\mathrm{Si}$, and mouth (jaw, lip, and tongue) region in monkeys $\mathrm{Hi}$ and $\mathrm{Ch}$ (Table 1). The injection sites together with the results of ICMS mapping were indicated in Fig. 2 (see also Supplementary Fig. 1).

\section{Histological Procedures}

Four days after the rabies injections, when the viral infection is expected to reach 3rd-order neurons, the monkeys were anesthetized deeply with an overdose of sodium pentobarbital (50 mg/kg, i.v.) and perfused transcardially with $0.1 \mathrm{M}$ phosphate-buffered saline (PBS; pH 7.4), followed by a mixture of $10 \%$ formalin and $15 \%$ saturated picric acid in $0.1 \mathrm{M}$ phosphate buffer $(\mathrm{pH}$ 7.4). The brains were removed from the skull, post-fixed in the same fresh fixative overnight at $4^{\circ} \mathrm{C}$, saturated with $30 \%$ sucrose at $4^{\circ} \mathrm{C}$, and then cut serially into coronal sections $60 \mu \mathrm{m}$ thick on a freezing microtome. Every sixth section was immunostained with anti-rabies antibody raised in rabbit (Inoue et al., 2003) and anti-rabbit IgG antibody raised in goat (Vector laboratories, Burlingame, CA, USA) by means of the avidin-biotin-peroxidase complex (ABC) method. A series of the adjacent sections (60 $\mu \mathrm{m}$ apart) were Nissl-stained with $1 \%$ Neutral Red or Cresyl Violet. 
The detailed procedures were as described in our previous papers (Miyachi et al., 2005, 2006).

\section{Data Analysis}

Neuronal labeling in the prefrontal cortex was plotted on tracings of representative coronal sections. The unfolded maps were reconstructed from sections $360 \mu \mathrm{m}$ apart (in monkeys X, Su, and Hi; Figs. 3, 4, 5) or sections $720 \mu \mathrm{m}$ apart (in monkeys Lo, Si, and Ch; Supplementary Fig. 1). All unfolded lines were aligned rostrally at the fundus of the principal sulcus and caudally at the genu of the arcuate sulcus (for detail, see Barbas and Pandya, 1987; Hatanaka et al., 2001).

Delineation of the VLPF was made according to the cytoarchitrectonic criteria described by Gerbella et al. (2007) and Borra et al. (2011), whereas the other prefrontal sectors were divided according to Barbas and Pandya (1989) (Fig. 1). 


\section{Results}

Rabies injection sites

Prior to rabies virus injections, body-part representations of the MI were determined by means of ICMS. The stimulating parameters (see Materials and Methods) were comparable to those previously used for mapping of the MI, but much milder than those for premotor cortical mapping (Godschalk et al., 1995; Takada et al., 1998; Hatanaka et al., 2005). On stimulation of the arm and hand regions, brief movements of the shoulder, elbow, wrist, or digit(s) were induced by currents lower than $20 \mu \mathrm{A}$ in most cases. Digit movements were often elicited at $<10 \mu \mathrm{A}$. On stimulation of the mouth region, twitches of the tongue or lip or brief jaw movements were frequently induced by currents lower than $20 \mu \mathrm{A}$. Cytoarchitectonic analysis revealed that the cortex around the sites of rabies injections in the MI contains giant pyramidal neurons in layer V. Incipient granular layer IV, which is a characteristic of the ventral area 6 immediately rostral to the MI (Barbas and Pandya, 1987), was not observed (Fig. 2B).

Retrograde transneuronal labeling in the prefrontal cortex

We judged that rabies labeling in all cases reached third-order neurons, because a number 
of striatal projection neurons were labeled by way of the thalamus (containing first-order neurons) and the internal pallidal segment (containing second-order neurons) along the cortico-basal ganglia loop. Only in one monkey (monkey $\mathrm{Ch}$ ), striatal interneurons were also labeled. This indicated that the rabies labeling in this monkey proceeded at least partly to fourth-order neurons (see Miyachi et al., 2006).

In all cases, the injection sites, as well as the cortical areas that are considered to connect to the injection sites monosynaptically or disynaptically (e.g., the caudal and rostral premotor areas), were filled with a tremendous number of the labeled neurons (Figs. 3A, 4A, 5A; for the injection site, see Fig. 2B).

Neuronal labeling from the MI arm region

In two monkeys ( $\mathrm{X}$ and Lo), rabies virus was injected into the MI arm region representing the shoulder and elbow (Fig. 2C; see also Supplementary Fig. 1). Four days after the rabies injections, labeled neurons were found in the medial, lateral, and orbital sectors of the prefrontal cortex. Overall, neuronal labeling from the arm region (Fig. 3; see also Supplementary Fig. 1) was so sparse as compared to that from the entire forelimb region (see Miyachi et al., 2005). Dense accumulation of labeled neurons in the VLPF, as seen 
after rabies injections into the entire forelimb region, was not observed. Lateral area 12

(area 12l) and adjacent area 45B contained more labeled neurons than other prefrontal areas, and few neurons were labeled in the dorsal bank of the principal sulcus (Fig. 3A, E-G, J-L). On the medial wall, most of the labeled neurons were found around the cingulate sulcus and in medial area 9, whereas the ventral part (areas 14 and 25) was largely devoid of neuronal labeling.

Neuronal labeling from the MI hand region

In two monkeys ( $\mathrm{Su}$ and $\mathrm{Si}$ ), rabies injections were made into the MI hand region representing the wrist and digits (Fig. 2C; see also Supplementary Fig. 1). Four days after the rabies injections, labeled neurons in the prefrontal cortex was much more abundant than in the arm-injection case (Fig. 4; see also Supplementary Fig. 1). In contrast to the arm-injection case, marked accumulation of labeled neurons was observed in several prefrontal areas. In areas 121 and 45B, the density of labeled neurons was increased considerably as compared to the arm-injection case, and the area of dense neuron labeling was extended into area 12o and the caudal part of area 45B (Fig. 4F, G, K, L). In these areas, labeled neurons were more abundant in the deeper layers. Conspicuous neuron 
labeling was observed in the ventral lip of the principal sulcus at its rostrocaudal middle level, and the adjacent area in the ventrolateral convexity. This dense neuronal labeling resided primarily in area 46v and slightly extended into adjacent area 12r (Gerbella, 2007; Borra, 2011) (Fig. 4A, B-D, I, J; see also Supplementary Fig. 1). The labeled neurons were evenly distributed in the superficial and deep layers. Such dense clustering of labeled neurons was not observed in adjacent area 45A (Fig. 4E-G, K, L). In the DLPF, a relatively small but distinct cluster of labeled neurons was located in the dorsal lip of the principal sulcus, at a more caudal level than the dense labeling zone in the ventral lip of the sulcus (Fig. 4A, E, L). In addition, area 9 (both medial and lateral parts) and area 24 around the cingulate sulcus contained a number of labeled neurons (Fig. 4B-D, I, J). Like the case of arm injection, the ventral part of the medial wall (areas 14 and 25) and the dorsal bank of the principal sulcus (area 46d) were essentially devoid of neuronal labeling, except a distinct cluster of labeled neurons in the caudal part of area 46d, as described above. The overall pattern of prefrontal labeling was quite similar to that for the entire forelimb region of the MI (Miyachi et al., 2005), though the neuronal labeling in the VLPF was more prominent in the hand-injection case. 
Neuronal labeling from the MI mouth region

In two monkeys ( $\mathrm{Hi}$ and $\mathrm{Ch}$ ), rabies virus was injected into the MI mouth region representing the jaw, lip, and tongue (Fig. 2C; see also Supplementary Fig. 1). The prefrontal cortex of monkey Ch contained a much larger number of labeled neurons than in monkey Hi (Fig. 5; see also Supplementary Fig. 1), although the distribution pattern of the labeled neurons was quite similar in both monkeys. This was probably ascribable to an advanced stage of rabies infection in monkey $\mathrm{Ch}$ that allowed the fourth-order neuron labeling (for detail, see Retrograde transneuronal labeling in the prefrontal cortex).

The overall pattern of neuronal labeling was similar to that in the hand-injection case, with some differences. In contrast to the hand-injection case, the area of dense neuron labeling was largely confined to area 121 and only slightly extended into areas $45 \mathrm{~B}$ and 12o (Fig. 5A, E, F, G). A tremendous number of labeled neurons were accumulated in the ventral lip of the principal sulcus. As observed in the hand-injection case, this accumulation of labeled neurons was located mainly in the ventral part of area 46v with slight spread into adjacent area 12r (Fig. 5A, B-F, I-K; see also Supplementary Fig. 1). As seen in the hand-injection case, adjacent area 45A contained only a small number of labeled neurons. In the OF, a large number of labeled neurons were observed between the 
medial and the lateral orbital sulcus, corresponding to area 13 (Barbas and Pandya, 1989)

(Fig. 5A, F, G). In monkey Ch, this orbital labeling further spread into area 11. On the other hand, the dorsal bank of the principal sulcus contained only a few labeled neurons, except for its caudal level. On the medial wall, like in the arm- and hand-injection cases, most of the labeled neurons were found around the cingulate sulcus and in medial area 9. The ventral part of the medial wall (areas 14 and 25) was virtually devoid of neuronal labeling. Dense neuronal labeling was seen in medial area 9 of monkey $\mathrm{Ch}$, but not of monkey Hi (compare Fig. 5C, D, J, K). 


\section{Discussion}

Using retrograde tanssynaptic transport of rabies virus, we investigated the organization of multisynaptic projections from the prefrontal cortex to different body-part representations of the MI. In each monkey, rabies injections were made into an MI region representing the arm, hand, or mouth that had been identified by means of ICMS mapping. The monkeys were sacrificed at the 4-day postinjection period when viral infection was expected to reach the prefrontal cortex retrogradely, resulting in the third-order neuron labeling (Kelly and Strick, 2000; Miyachi et al., 2005; Lu et al., 2007; Ninomiya et al., 2011). The third-order labeling was confirmed by examining neuronal labeling in the basal ganglia in all but one monkey. In monkey $\mathrm{Ch}$, a small number of the fourth-order neurons (i.e., interneurons in the striatum) were rabies-labeled (Miyachi et al., 2006). In the other monkeys, rabies labeling was observed in striatal projection neurons, but not in interneurons innervating the projection neurons, which indicates that the transneuronal labeling reached third-order neurons, but not fourth-order neurons.

The distribution pattern of rabies-labeled neurons was considerably different depending on the injection site in the MI. After the rabies injections into the hand or mouth representation, dense neuron labeling was observed in the ventral lip of the 
principal sulcus at its rostrocaudal middle level, and the adjacent area in the ventrolateral convexity. The labeled neurons in the same area were much fewer after the injections into the arm region. Cytoarchitectonically, this area includes the surface region of area $46 \mathrm{v}$ with slight extension into area 12r (Carmichael and Price, 1994; Borra et al., 2011), and corresponds probably to area 9/46v of Petrides and Pandya (1999). This prefrontal area is known to have strong connections with the ventral premotor area and inferior parietal cortex (Petrides and Pandya, 2001; Borra et al., 2011, Gerbella et al., 2012), which are important for motor actions by the hand and mouth. Accordingly, Luppino and his colleagues proposed that this area of the prefrontal cortex is involved in goal-directed hand and mouth actions. Our present data are in good accordance with this proposal.

The rabies injections into the mouth region of the MI produced dense neuronal labeling in an OF area located between the medial and the lateral orbital sulcus, corresponding to area 13 (Barbas and Pandya, 1989). This labeling pattern was not observed after the rabies injections into the MI hand region. The OF including area 13 receives sensory inputs with various modalities, such as olfaction and gustation (Saleem et al., 2008; for review, Cavada, 2000), and is thought to be important for assessment of objects, especially food. In favor of this, electrophysiological studies have reported that 
neurons in this area responded to food/liquid reward and sensory cues preceding the food/liquid reward (Tremblay and Schultz, 1999, 2000; Hikosaka and Watanabe, 2000, 2004; Bouret and Richmond, 2010). Therefore, it would be reasonable if the OF sends such information preferentially to the mouth region of the MI.

The present results have revealed the existence of strong trisynaptic projections from the ventral lip of the principal sulcus (area 46v) to the hand and mouth representations of the MI, and from the orbitofrontal cortex to the mouth region of the MI. These data suggest that the hand and mouth regions have a privileged position to receive a set of cognitive information from the VLPF (and OF for the mouth region). However, the transneuronal tracing technique does not give much information about which cortical areas or subcortical regions would mediate such multisynaptic projections. The projections are most likely to be mediated by multiple parallel routes. Several possible routes may be suggested. The projections from area $46 \mathrm{v}$ to the MI hand and mouth regions are likely relayed through the rostral and then the caudal part of the ventral premotor cortex (Matelli et al., 1986; Tokuno and Tanji, 1993; Hatanaka, et al., 2005; Borra et al., 2011; Gerbella et al., 2011; 2012), and/or the rostral and then the caudal cingulate motor areas (Dum and Strick, 1991; Hatanaka et al., 2003; Takada et al., 2004; 
Morecraft et al., 2012). In addition, the projections may be mediated through the AIP and then the ventral premotor cortex (Tokuno and Tanji, 1993; Luppino et al., 1999; Hatanaka, et al., 2005). Besides area 46v, areas 12l, 45B, and the dorsal lip of the principal sulcus at its caudal level were also strongly labeled from the MI hand region. These areas are known to send output projections to area F7 that is located in the rostral part of the dorsal premotor cortex (Luppino et al., 2003). Therefore, the projections from these prefrontal areas would probably be mediated by the rostral and caudal parts of the dorsal premotor cortex. Regarding the projectinons from the orbitofrontal cortex to the MI mouth region, previous studies have shown that the rostral part of the insular cortex receives input from area 13 (Saleem et al., 2008) and, in turn, projects to the principal part of the cortical masticatory area in the ventral premotor cortex (Hatanaka et al., 2005). This may probably be the main pathway linking area 13 to the mouth region of the MI.

Together with the data from our previous study (Miyachi et al., 2005), the present results suggest that the MI regions representing different body parts receive distinct patterns of the prefrontal inputs. Especially, the hand and mouth regions, engaged in the most elaborate motor actions, preferentially receive inputs from specific regions of the VLPF and/or OF. 


\section{Acknowledgements}

We are grateful to M. Imanishi, Y. Ito, T. Kuroda, and E. Mine for technical assistance.

We thank Drs. N. Hatanaka and D. Takahara for valuable comments and discussions. This work was supported by a Grant-in-Aid for Scientific Research on Priority Areas (Integrative Brain Research) from the Ministry of Education, Culture, Sports, Science and Technology of Japan (17021050 to M.T.). The entire experiments of this work were conducted at the Tokyo Metropolitan Institute for Neuroscience, Tokyo Metropolitan Organization for Medical Research (recently reorganized to the Tokyo Metropolitan Institute of Medical Science), Tokyo, Japan. 


\section{References}

Barbas, H., 1988. Anatomic organization of basoventral and mediodorsal visual recipient prefrontal regions in the rhesus monkey. J Comp Neurol. 276, 313-342.

Barbas, H., 1993. Organization of cortical afferent input to orbitofrontal areas in the rhesus monkey. Neuroscience. 56, 841-864.

Barbas, H., Mesulam, M.M., 1985. Cortical afferent input to the principalis region of the rhesus monkey. Neuroscience. 15, 619-637.

Barbas, H., Pandya, D.N., 1987. Architecture and frontal cortical connections of the premotor cortex (area 6) in the rhesus monkey. J Comp Neurol. 256, 211-228.

Barbas, H., Pandya, D.N., 1989. Architecture and intrinsic connections of the prefrontal cortex in the rhesus monkey. J Comp Neurol. 286, 353-375.

Borra, E., Gerbella, M., Rozzi, S., Luppino, G.,2011. Anatomical evidence for the involvement of the macaque ventrolateral prefrontal area $12 \mathrm{r}$ in controlling goal-directed actions. J Neurosci. 31, 12351-12363.

Bouret, S., Richmond, B.J., 2010. Ventromedial and orbital prefrontal neurons differentially encode internally and externally driven motivational values in monkeys. J Neurosci. 30, 8591-8601. 
Carmichael, S.T., Price, J.L., 1994. Architectonic subdivision of the orbital and medial prefrontal cortex in the macaque monkey. J Comp Neurol. 346, 366-402.

Carmichael, S.T., Price, J.L.,1995a. Limbic connections of the orbital and medial prefrontal cortex in macaque monkeys. J Comp Neurol. 363, 615-641.

Carmichael, S.T., Price, J.L., 1995b. Sensory and premotor connections of the orbital and medial prefrontal cortex of macaque monkeys. J Comp Neurol. 363, 642-664.

Cavada, C., Company, T., Tejedor, J., Cruz-Rizzolo, R.J., Reinoso-Suarez, F., 2000. The anatomical connections of the macaque monkey orbitofrontal cortex. A review. Cereb Cortex. 10, 220-242.

di Pellegrino, G., Wise, S.P., 1991. A neurophysiological comparison of three distinct regions of the primate frontal lobe. Brain. 114, 951-978.

Dum, R.P., Strick, P.L., 1991. The origin of corticospinal projections from the premotor areas in the frontal lobe. $J$ Neurosci. 11, 667-689.

Gerbella, M., Belmalih, A., Borra, E., Rozzi, S., Luppino, G., 2007. Multimodal architectonic subdivision of the caudal ventrolateral prefrontal cortex of the macaque monkey. Brain Struct Funct. 212, 269-301. 
Gerbella, M., Belmalih, A., Borra, E., Rozzi, S., Luppino, G., 2010. Cortical connections of the macaque caudal ventrolateral prefrontal areas 45A and 45B. Cereb Cortex. 20, 141-168.

Gerbella, M., Belmalih, A., Borra, E., Rozzi, S., Luppino, G., 2011. Cortical connections of the anterior (F5a) subdivision of the macaque ventral premotor area F5. Brain Struct Funct. 216, 43-65.

Gerbella, M., Borra, E., Tonelli, S., Rozzi, S., Luppino, G., 2012. Connectional Heterogeneity of the Ventral Part of the Macaque Area 46. Cereb Cortex. In press (first published online April 11, 2012 doi:10.1093/cercor/bhs096).

Godschalk, M., Mitz, A.R., van Duin, B., van der Burg, H., 1995. Somatotopy of monkey premotor cortex examined with microstimulation. Neurosci Res. 23, 269-279.

Hatanaka, N., Nambu, A., Yamashita, A., Takada, M., Tokuno, H., 2001. Somatotopic arrangement and corticocortical inputs of the hindlimb region of the primary motor cortex in the macaque monkey. Neurosci Res. 40, 9-22.

Hatanaka, N., Tokuno, H., Hamada, I., Inase, M., Ito, Y., Imanishi, M., Hasegawa, N., Akazawa, T., Nambu, A., Takada, M., 2003. Thalamocortical and intracortical connections of monkey cingulate motor areas. J Comp Neurol. 462, 121-138. 
Hatanaka, N., Tokuno, H., Nambu, A., Inoue, T., Takada, M., 2005. Input-output organization of jaw movement-related areas in monkey frontal cortex. J Comp Neurol. 492, 401-425.

Hikosaka, K., Watanabe, M., 2000. Delay activity of orbital and lateral prefrontal neurons of the monkey varying with different rewards. Cereb Cortex. 10, 263-271.

Hikosaka, K., Watanabe, M., 2004. Long- and short-range reward expectancy in the primate orbitofrontal cortex. Eur J Neurosci. 19, 1046-1054.

Hoshi, E., Shima, K., Tanji, J., 1998. Task-dependent selectivity of movement-related neuronal activity in the primate prefrontal cortex. J Neurophysiol. 80, 3392-3397.

Hoshi, E., Shima, K., Tanji, J., 2000. Neuronal activity in the primate prefrontal cortex in the process of motor selection based on two behavioral rules. J Neurophysiol. $\mathbf{8 3}$, $2355-2373$.

Inoue, S., Sato, Y., Hasegawa, H., Noguchi, A., Yamada, A., Kurata, T., Iwasaki, T., 2003. Cross-reactive antigenicity of nucleoproteins of lyssaviruses recognized by a monospecific antirabies virus nucleoprotein antiserum on paraffin sections of formalin-fixed tissues. Pathol Int. 53, 525-533. 
Kelly, R.M., Strick, P.L., 2000. Rabies as a transneuronal tracer of circuits in the central nervous system. J Neurosci Methods. 103, 63-71.

Lu, X., Miyachi, S., Ito, Y., Nambu, A. Takada, M., 2007. Topographic distribution of output neurons in cerebellar nuclei and cortex to somatotopic map of primary motor cortex. Eur J Neurosci. 25, 2374-2382.

Luppino, G., Murata, A., Govoni, P., Matelli, M., 1999. Largely segregated parietofrontal connections linking rostral intraparietal cortex (areas AIP and VIP) and the ventral premotor cortex (areas F5 and F4). Exp Brain Res. 128, 181-187.

Luppino, G., Rozzi, S., Calzavara, R., Matelli, M, 2003. Prefrontal and agranular cingulate projections to the dorsal premotor areas F2 and F7 in the macaque monkey. Eur J Neurosci. 17, 559-578.

Matelli, M., Camarda, R., Glickstein, M. Rizzolatti, G., 1986. Afferent and efferent projections of the inferior area 6 in the macaque monkey. J Comp Neurol. 251, 281-298.

Miller, E.K., Erickson, C.A., Desimone, R., 1996. Neural mechanisms of visual working memory in prefrontal cortex of the macaque. J Neurosci. 16, 5154-5167. 
Mishkin, M., Manning, F.J., 1978. Non-spatial memory after selective prefrontal lesions in monkeys. Brain Res. 143, 313-323.

Miyachi, S., Lu, X., Inoue, S., Iwasaki, T., Koike, S., Nambu, A., Takada, M., 2005. Organization of multisynaptic inputs from prefrontal cortex to primary motor cortex as revealed by retrograde transneuronal transport of rabies virus. $J$ Neurosci. 25, 2547-2556.

Miyachi, S., Lu, X., Imanishi, M., Sawada, K., Nambu, A., Takada, M., 2006. Somatotopically arranged inputs from putamen and subthalamic nucleus to primary motor cortex. Neurosci Res. 56, 300-308.

Morecraft, R.J., Stilwell-Morecraft, K.S., Cipolloni, P.B., Ge, J., McNeal, D.W., Pandya, D.N., 2012. Cytoarchitecture and cortical connections of the anterior cingulate and adjacent somatomotor fields in the rhesus monkey. Brain. 87, 457-497.

Ninomiya, T., Sawamura, H., Inoue, K.I., Takada, M., 2011. Differential Architecture of Multisynaptic Geniculo-Cortical Pathways to V4 and MT. Cereb Cortex. 21, 2797-2808.

Pandya, D.N., Yeterian, E.H., 1990. Prefrontal cortex in relation to other cortical areas in rhesus monkey: architecture and connections. Prog Brain Res. 85, 63-94. 
Passingham, R., 1975. Delayed matching after selective prefrontal lesions in monkeys (Macaca mulatta). Brain Res. 92, 89-102.

Petrides, M., Pandya, D.N., 1999. Dorsolateral prefrontal cortex: comparative cytoarchitectonic analysis in the human and the macaque brain and corticocortical connection patterns. Eur J Neurosci. 11, 1011-1036.

Petrides, M., Pandya, D.N., 2001. Comparative cytoarchitectonic analysis of the human and the macaque ventrolateral prefrontal cortex and corticocortical connection patterns in the monkey. Eur J Neurosci. 16, 291-310.

Saleem, K.S., Kondo, H., Price, J.L., 2008. Complementary circuits connecting the orbital and medial prefrontal networks with the temporal, insular, and opercular cortex in the macaque monkey. J Comp Neurol. 506, 659-693.

Takada, M., Tokuno, H., Nambu, A., Inase, M., 1998. Corticostriatal projections from the somatic motor areas of the frontal cortex in the macaque monkey: segregation versus overlap of input zones from the primary motor cortex, the supplementary motor area, and the premotor cortex. Exp Brain Res. 120, 114-128. 
Takada, M., Nambu, A., Hatanaka, N., Tachibana, Y., Miyachi, S., Taira, M., Inase, M., 2004. Organization of prefrontal outflow toward frontal motor-related areas in macaque monkeys. Eur J Neurosci. 19, 3328-3342.

Tokuno, H., Tanji, J., 1993. Input organization of distal and proximal forelimb areas in the monkey primary motor cortex: a retrograde double labeling study. J comp neurol. 333, 199-209.

Tremblay, L., Schultz, W., 1999. Relative reward preference in primate orbitofrontal cortex. Nature. 398, 704-708.

Tremblay, L., Schultz, W., 2000. Reward-related neuronal activity during go-nogo task performance in primate orbitofrontal cortex. J Neurophysiol. 83, 1864-1876.

Warden, M.R., Miller, E.K., 2007. The representation of multiple objects in prefrontal neuronal delay activity. Cereb Cortex. 17, i41-50.

Wilson, F.A., Scalaidhe, S.P., Goldman-Rakic, P.S., 1993. Dissociation of object and spatial processing domains in primate prefrontal cortex. Science. 260, 1955-1958. 
Figure legends

Figure 1. Parcellation of the prefrontal cortex on a map where the sulci are unfolded. The ventrolateral prefrontal cortex (areas 46v, 12r, 121, 12o, 45A, and 45B) was delineated according to the definitions by Borra et al. (2011), while the definitions of other prefrontal areas followed Barbas and Pandya (1989). CgS, cingulate sulcus; iAS, inferior limb of the arcuate sulcus; LOS, lateral orbital sulcus; MOS, medial orbital sulcus; PS, principal sulcus; sAS, superior limb of the arcuate sulcus.

Figure 2. Rabies injection sites in the MI. A. Approximate areas of viral injections in the arm, hand, and mouth representations were indicated schematically on the lateral view of the brain. B. Nissl-stained (left) and rabies-immunostained (right) sections through the rostralmost level of the injection site in monkey Hi (mouth-injection case). C. Results of ICMS mapping and the locations of the rabies injection sites (open circles) in the MI in three injection cases. CS, central sulcus; D, digits; E, elbow; Fa, face; J, jaw; L, Lip; N, neck; S, shoulder; Tg, tongue; Tr, trunk; W, wrist. 
Figure 3. Distribution of labeled neurons in the prefrontal cortex and the adjacent motor-related areas 4 days after rabies injections into the MI arm region (monkeys $\mathrm{X}$ and Lo). A. Surface-view reconstruction showing the distribution pattern of neuronal labeling in monkey X. Solid and thin dashed lines indicate sulci. Thick dashed lines denote the borders between the medial and the lateral surface, and between the lateral and the orbital surface. Four different sizes of dots represent the ranges of labeled neuron number. Abbreviations are as in Fig. 1. B-G. Distribution of labeled neurons in representative coronal sections of the frontal lobe. The rostrocaudal levels of the sections are specified by arrows in A. H-L. Distribution of rabies-labeled neurons in monkey Lo. Representative coronal sections (I-L) of which rostrocaudal levels are specified in the lateral view of the brain of $(\mathrm{H})$. Arrow heads indicate the borders between cortical areas.

Figure 4. Distribution of labeled neurons in the prefrontal cortex and the adjacent motor-related areas 4 days after rabies injections into the MI hand region (monkeys Su and $\mathrm{Si}$ ). A. Surface-view reconstruction showing the distribution pattern of neuronal labeling in monkey Su. IOS, intermediate orbital sulcus. Other abbreviations are as in Fig. 1. B-G. Distribution of labeled neurons in representative coronal sections of the frontal 
lobe. H-L. Distribution of rabies-labeled neurons in monkey Si. All conventions are as in Fig. 3.

Figure 5. Distribution of labeled neurons in the prefrontal cortex and the adjacent motor-related areas 4 days after rabies injections into the MI mouth region (monkeys Hi and $\mathrm{Ch}$ ). A. Surface-view reconstruction showing the distribution pattern of neuronal labeling in monkey Hi. Abbreviations are as in Figs. 1 and 3. B-G. Distribution of labeled neurons in representative coronal sections of the frontal lobe. H-L. Distribution of rabies-labeled neurons in monkey Ch. All conventions are as in Fig. 3.

Supplementary Figure 1. Distributions of labeled neurons in the prefrontal cortex and the adjacent motor-related areas 4 days after rabies injections into the arm (monkey Lo), hand (monkey $\mathrm{Si}$ ), and mouth (monkey $\mathrm{Ch}$ ) regions of the MI. Surface-view reconstructions showing the distribution patterns of neuronal labeling. Solid and thin dashed lines indicate sulci. Thick dashed lines denote the borders between the medial and the lateral surface, and between the lateral and the orbital surface. Four different sizes of dots represent the ranges of labeled neuron number. $\mathrm{CgS}$, cingulate sulcus; iAS, inferior limb 
of the arcuate sulcus; IOS, intermediate orbital sulcus; LOS, lateral orbital sulcus; MOS,

medial orbital sulcus; PS, principal sulcus; sAS, superior limb of the arcuate sulcus.

(Inset) Results of ICMS mapping and the locations of the rabies injection sites (open circles) in the MI. CS, central sulcus; D, digits; E, elbow; Ey, eye lid; J, jaw; L, lip; S, shoulder; Tg, tongue; Tr, trunk; W, wrist. 
Table 1. Summary of rabies injections into MI

\begin{tabular}{lllllll}
\hline Name & Species & Sex & BW $(\mathrm{kg})$ & Injection site & Survival (days) & Injection volume $(\mu \mathrm{l})$ \\
\hline $\mathrm{X}$ & $\mathrm{R}$ & $\mathrm{M}$ & 10 & arm & 4 & 4.5 \\
$\mathrm{Lo}$ & $\mathrm{J}$ & $\mathrm{F}$ & 8 & arm & 4 & 5.5 \\
$\mathrm{Su}$ & $\mathrm{R}$ & $\mathrm{M}$ & 7 & hand & 4 & 4 \\
$\mathrm{Si}$ & $\mathrm{J}$ & $\mathrm{F}$ & 5 & hand & 4 & 5 \\
$\mathrm{Hi}$ & $\mathrm{R}$ & $\mathrm{M}$ & 8 & mouth & 4 & 5.5 \\
$\mathrm{Ch}$ & $\mathrm{C}$ & $\mathrm{M}$ & 6 & mouth & 4 & 10 \\
\end{tabular}

BW, body weight; C, crab-eating monkey (Macaca fascicularis); F, female; J, Japanese monkey (Macaca fuscata); M, male; R, rhesus monkey (Macaca mulatta). 


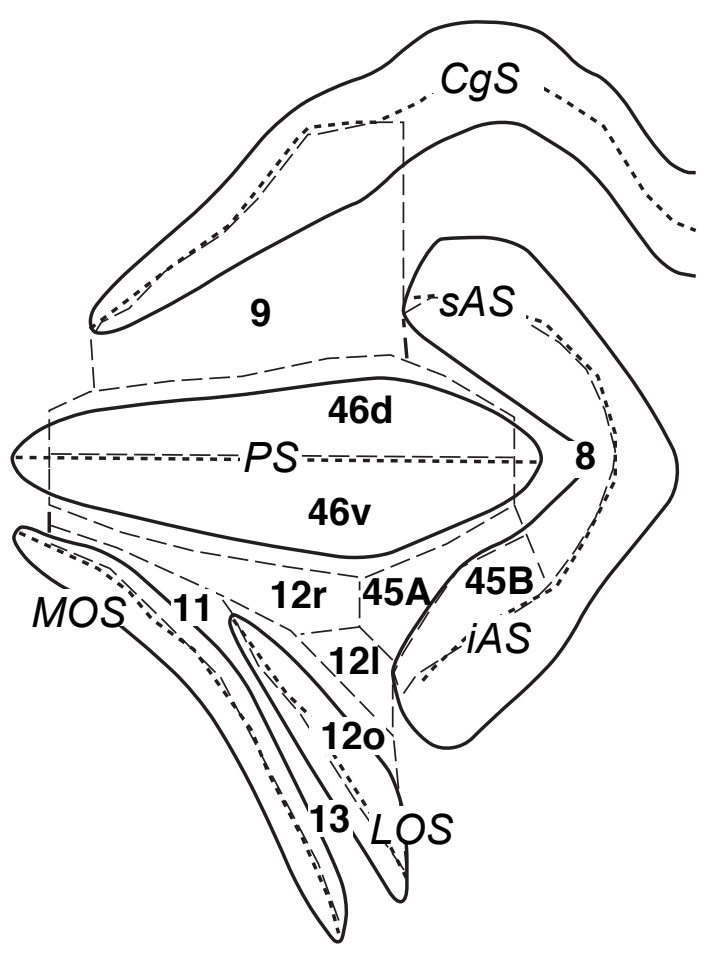

Figure 1

Miyachi et al. 
A.

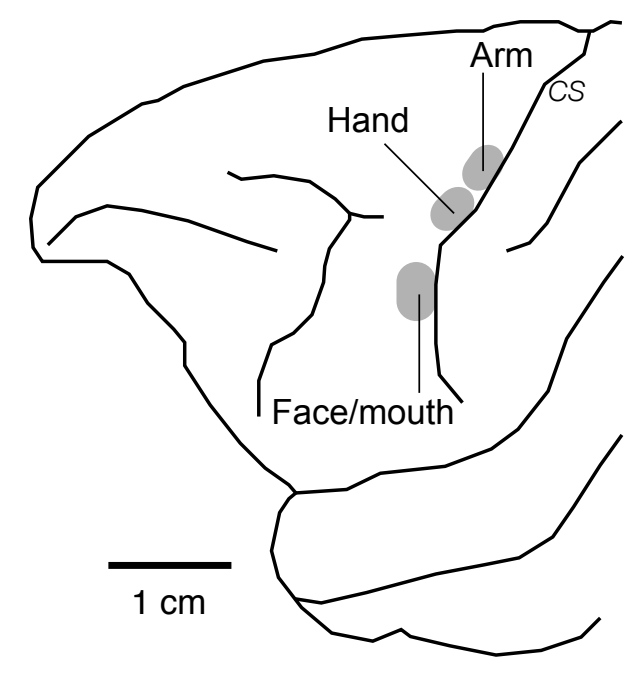

B.

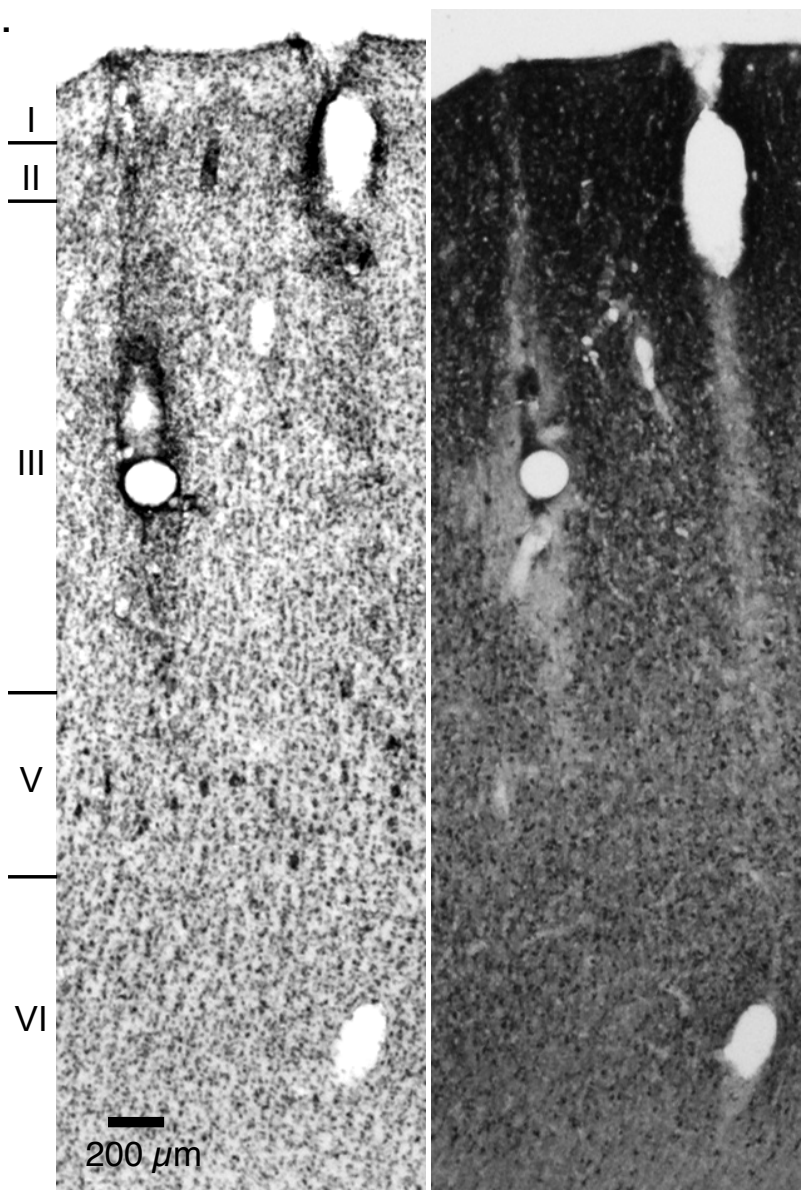

C.



Hand

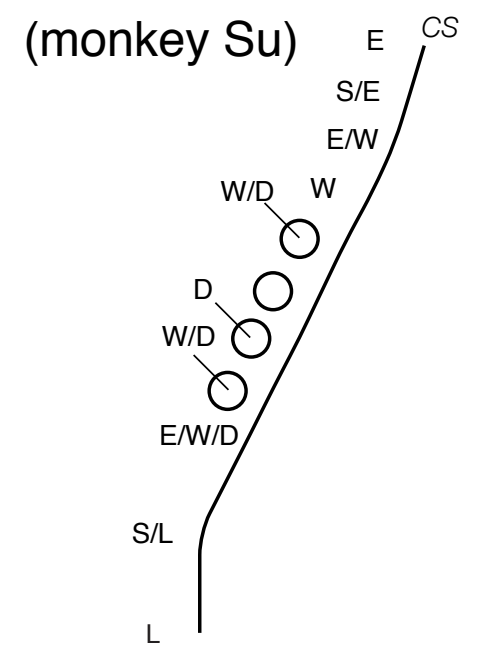

Mouth

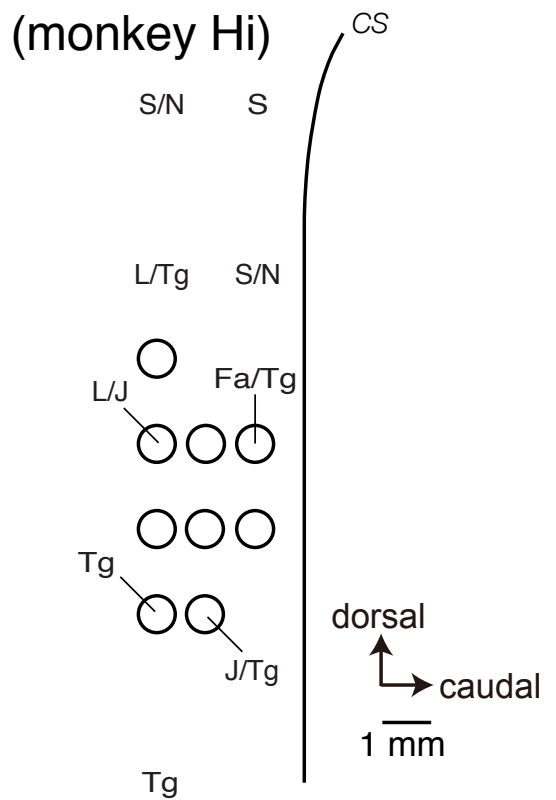

Figure 2

Miyachi et al. 


\section{MI arm}

\section{(monkey X)}

A
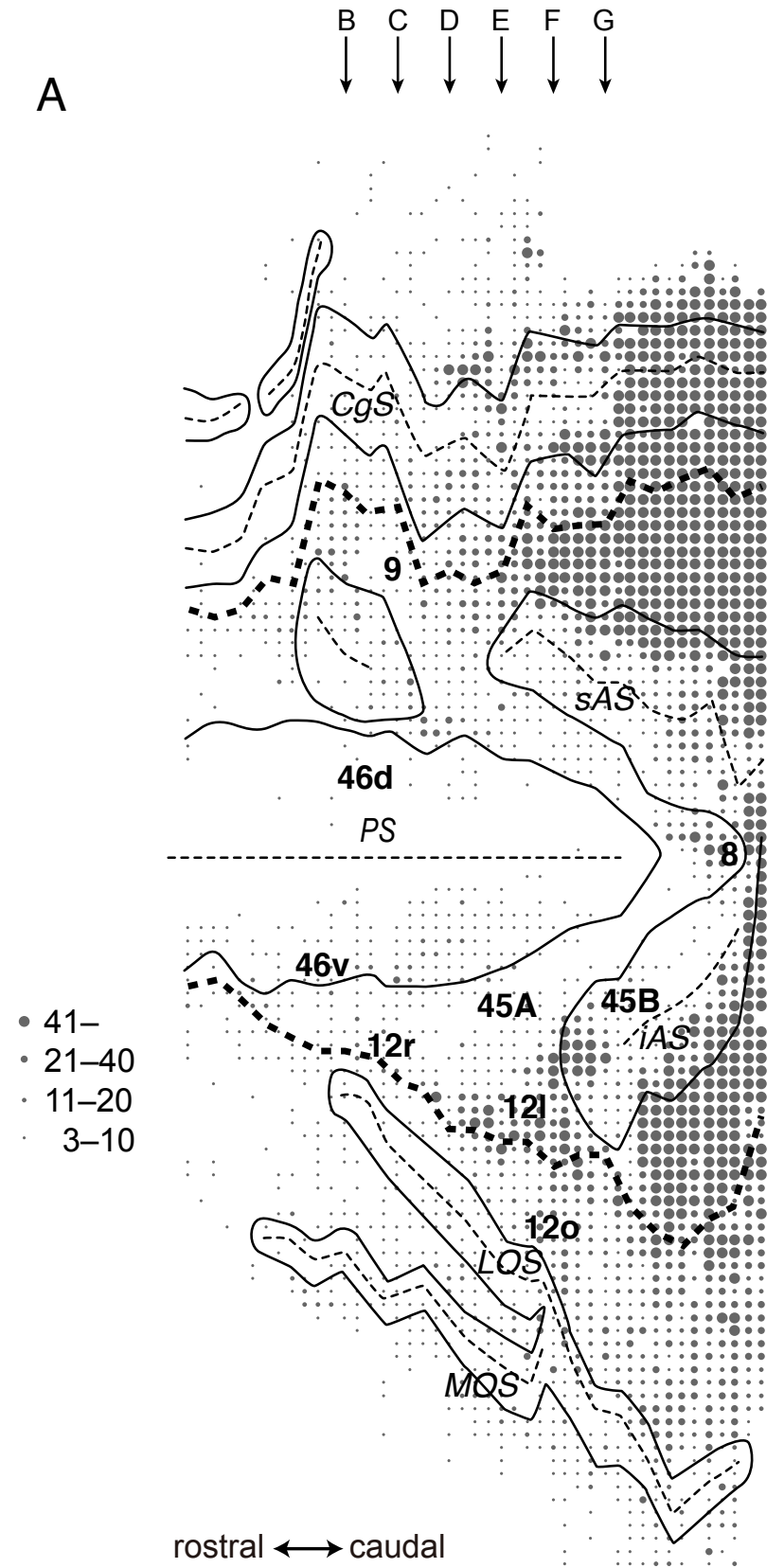

B
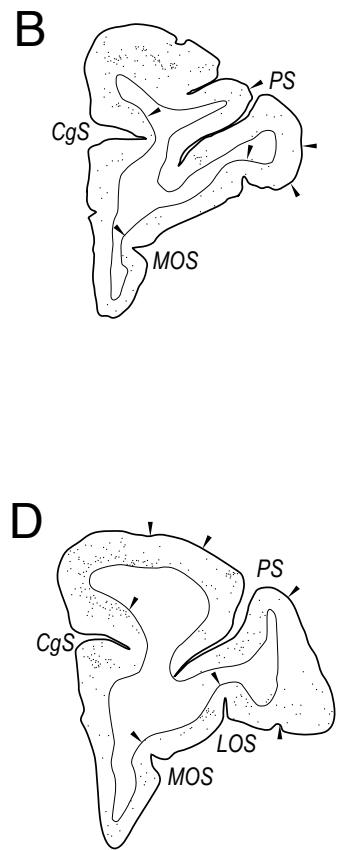

$\mathrm{F}$

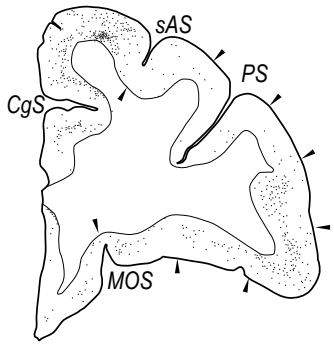

C

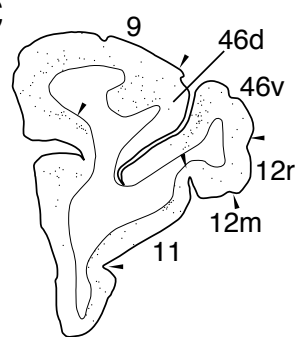

E

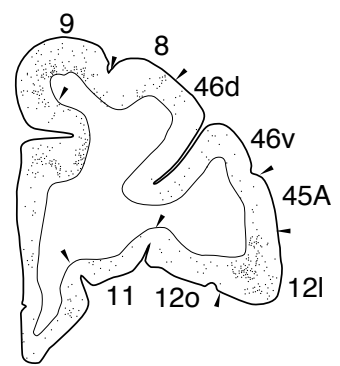

G

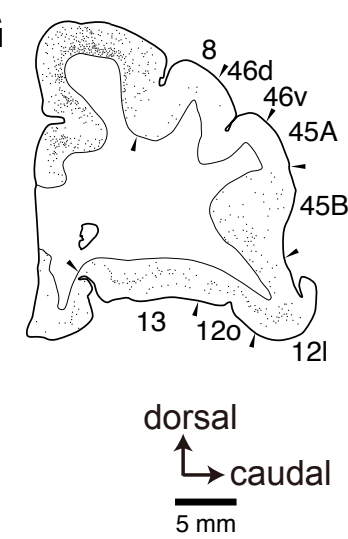

(monkey Lo)
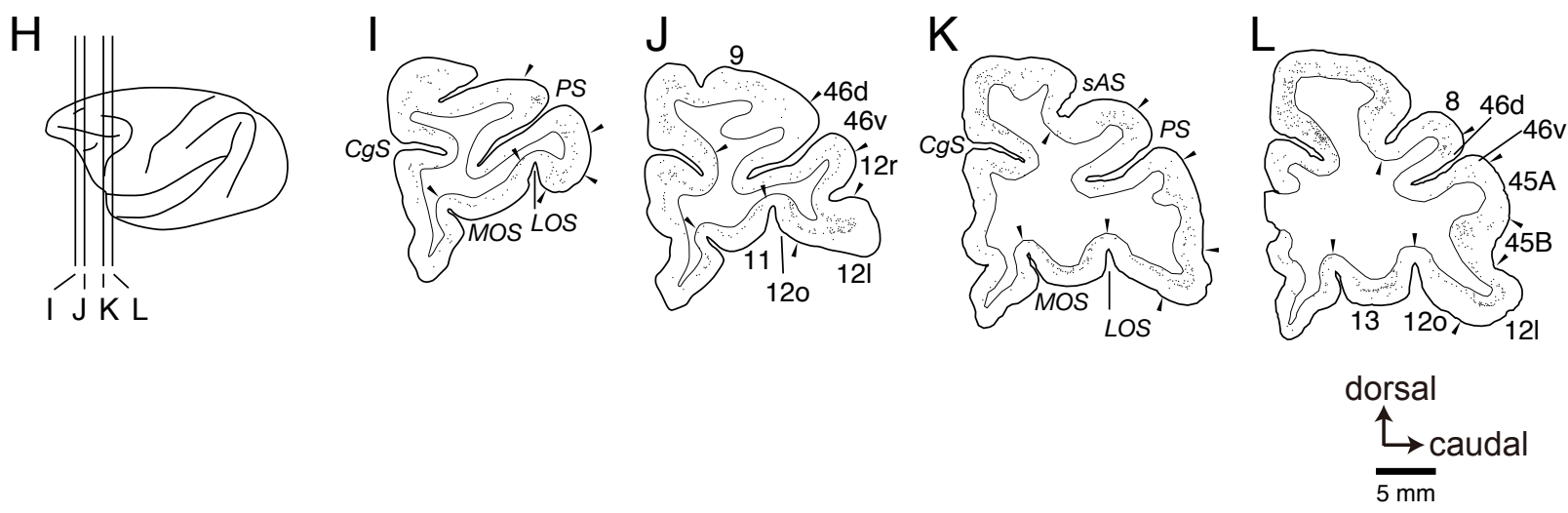

Figure 3

Miyachi et al. 
MI hand

(monkey Su)

A

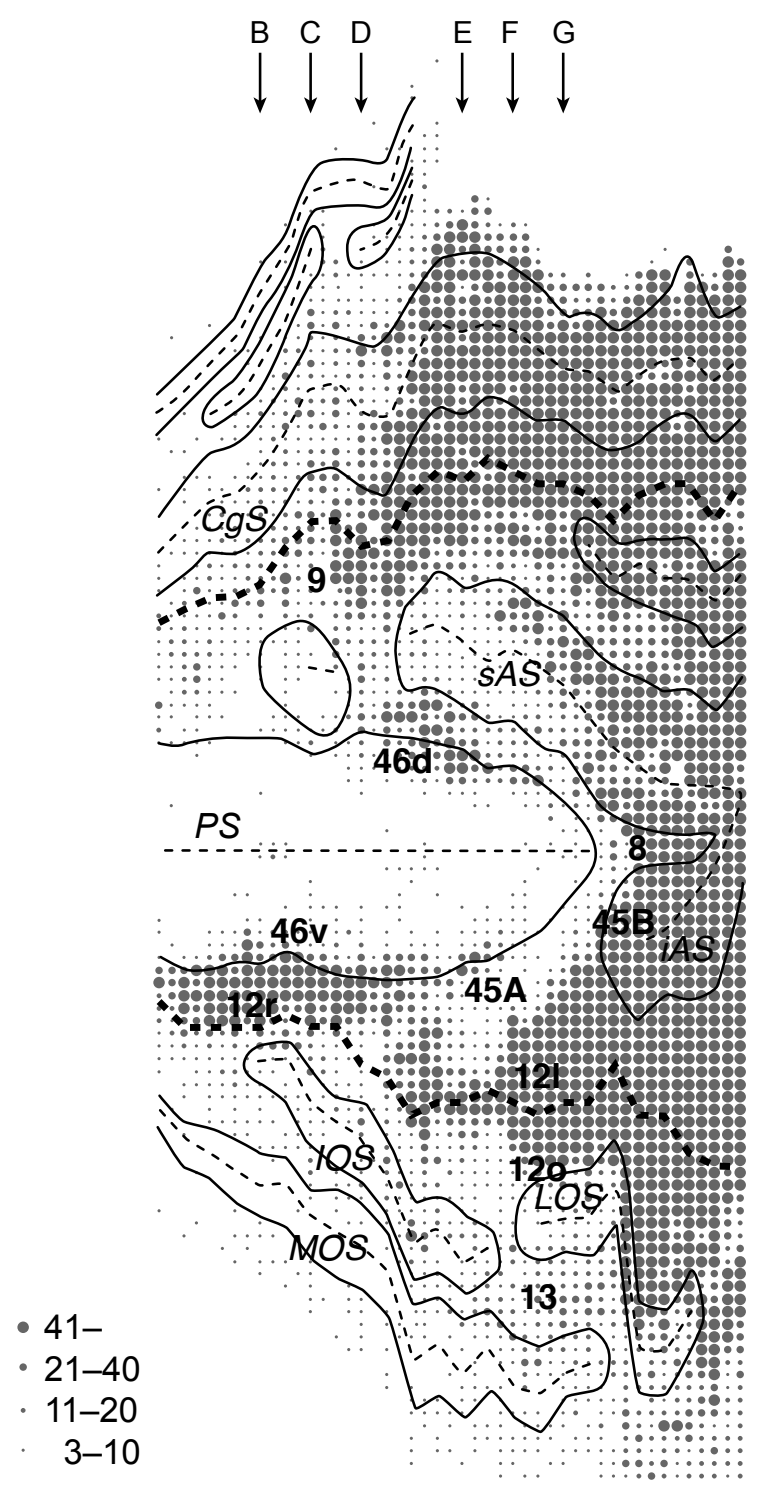

B
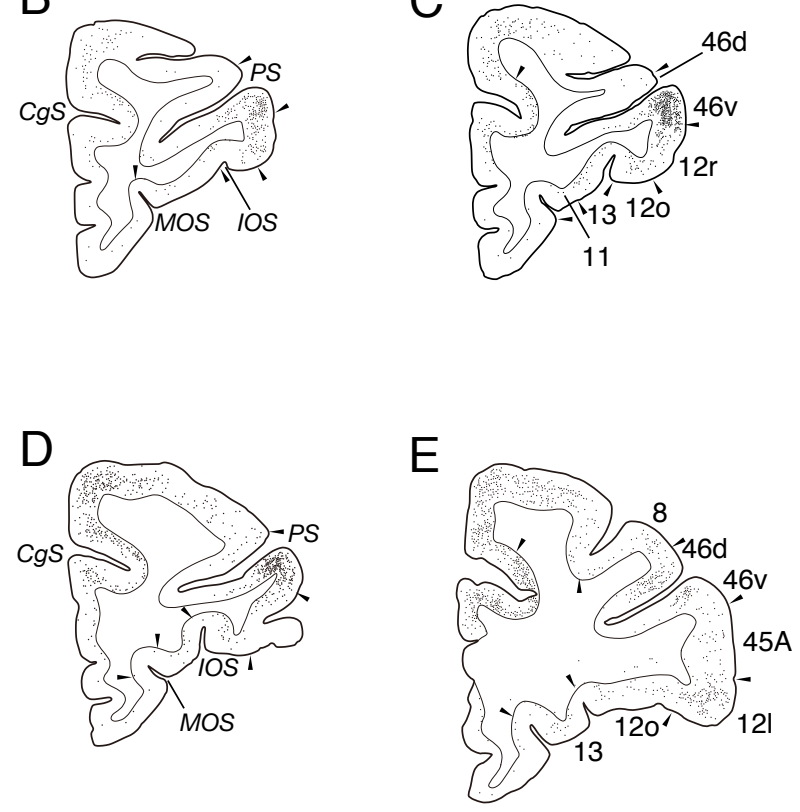

$\mathrm{F}$



E

C

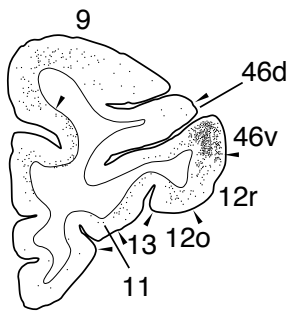

G

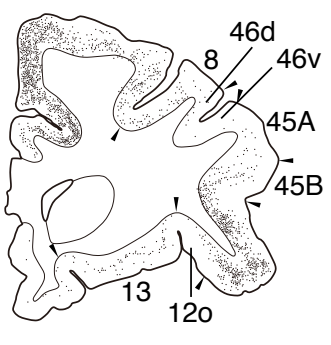

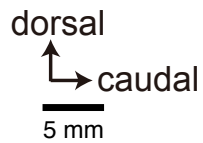

rostral $\longleftrightarrow$ caudal

\section{(monkey Si)}
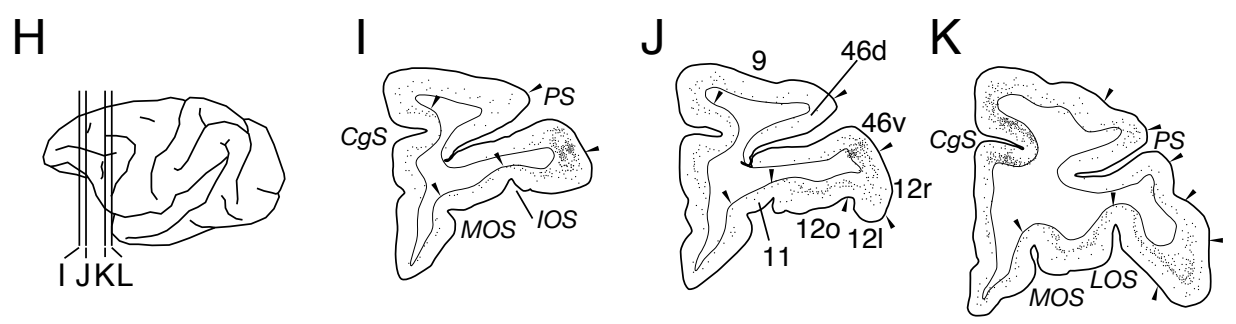

L

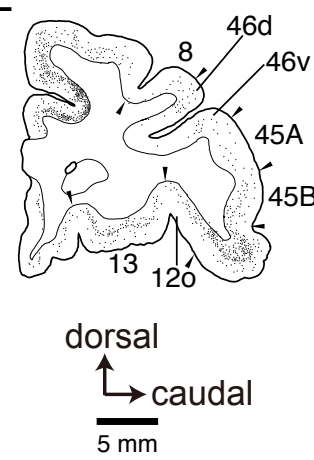

Figure 4

Miyachi et al. 
MI mouth

(monkey Hi)

A

$\begin{array}{llllll}B & C & D & E & F & G \\ \downarrow & \downarrow & \downarrow & \downarrow & \downarrow & \downarrow\end{array}$

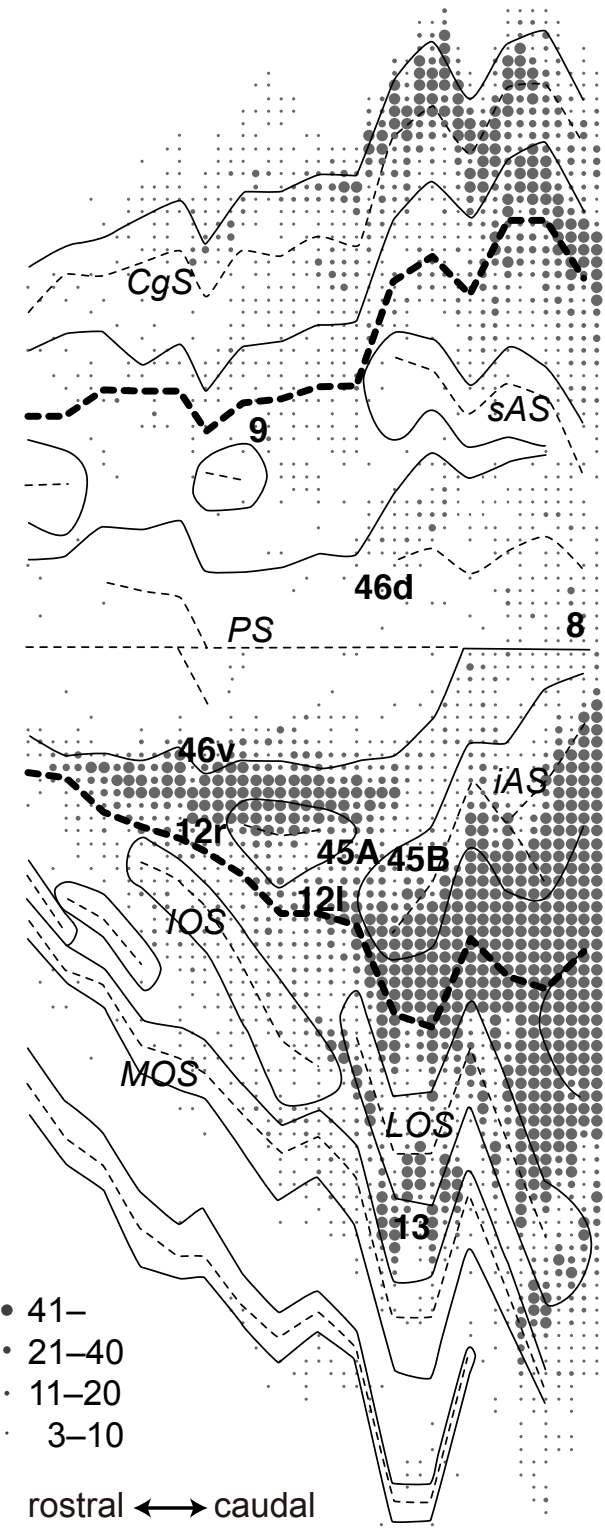

B

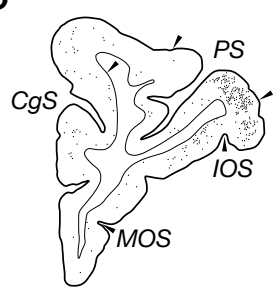

D

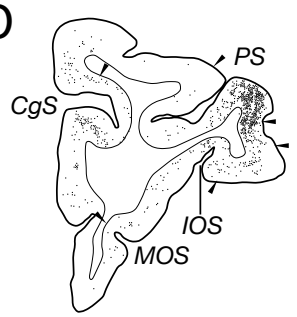

$\mathrm{F}$

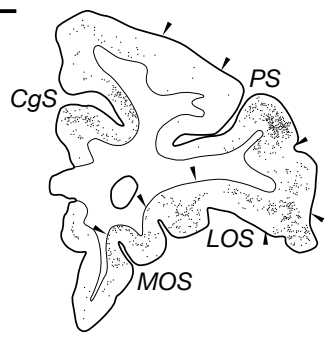

C

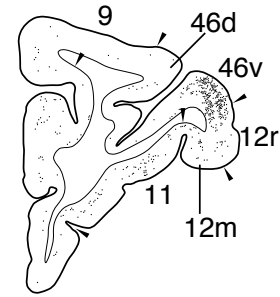

E

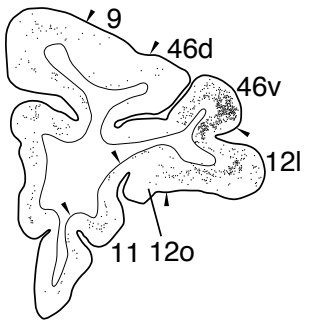

G

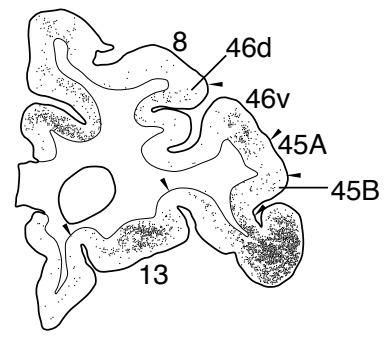

(monkey Ch)

$\mathrm{H}$

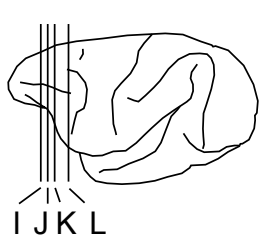

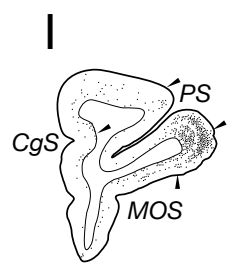

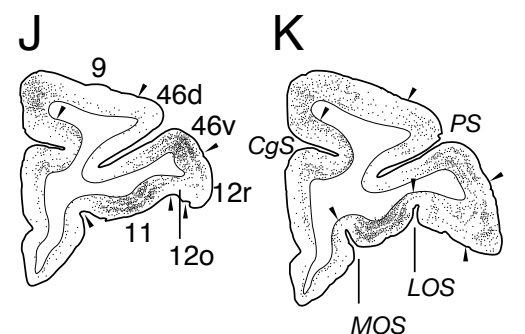

$\mathrm{L}$

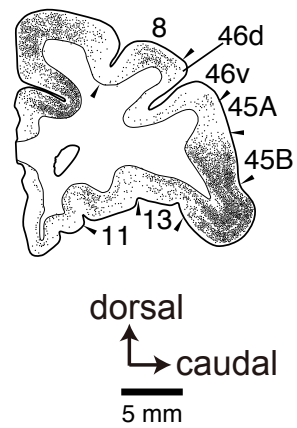

Figure 5

Miyachi et al. 
